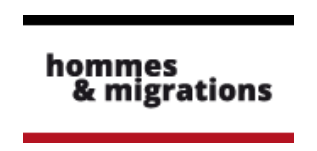

\section{Hommes \& migrations}

Revue française de référence sur les dynamiques

migratoires

$1281 \mid 2009$

France-Brésil sous l'angle des migrations et de l'altérité

\title{
Migrations et stratégies familiales dans les régions d'agrobusiness
}

Moacir Palmeira et Beatriz M. A. de Heredia

\section{(2) OpenEdition \\ 1 Journals}

Édition électronique

URL : http://journals.openedition.org/hommesmigrations/397

DOI : 10.4000/hommesmigrations.397

ISSN : 2262-3353

Éditeur

Musée national de l'histoire de l'immigration

Édition imprimée

Date de publication : 1 septembre 2009

Pagination : 142-153

ISSN : 1142-852X

\section{Référence électronique}

Moacir Palmeira et Beatriz M. A. de Heredia, « Migrations et stratégies familiales dans les régions

d'agrobusiness », Hommes \& migrations [En ligne], 1281 | 2009, mis en ligne le 29 mai 2013, consulté le 19 avril 2019. URL : http://journals.openedition.org/hommesmigrations/397 ; DOI : 10.4000/

hommesmigrations.397 


\section{Migrations et stratégies familiales dans les régions d’agrobusiness}

Par Moacir Palmeira, prof. PPGAS / Musée National / UFRJ et chercheur au CNPq et Beatriz M. A. de Heredia, prof. PPGSA / IFCS / UFRJ et chercheur au CNPq

Au Brésil, le développement de l'agrobusiness va de pair avec une forte croissance démographique. L'enquête a été menée dans la zone de production de soja autour de la route BR-163, au Mato Grosso, et celle du café do cerrado, dans le triangle Mineiro/Alto Paranaíba, au Minas Gerais. Mobilisée pour répondre aux besoins de main-d'œuvre, l'immigration interrégionale redessine les clivages au sein de la population

de ces territoires. Dans ces espaces de compétition économique, entre gaúchos et maranhenses, ou paulistas et baianos, les rapports de force se redistribuent en fonction du lieu de naissance des migrants. 
Les programmes gouvernementaux d'occupation des cerrados mis en ceuvre, avec quelques variations dans plusieurs États, durant les années soixante-dix, introduisent le soja, produit stratégique du projet, dans l'État du Goiás, du Minas Gerais (ouest et nord, outre le triangle Mineiro), de Bahia (ouest), du Maranhão (sud) et du Mato Grosso, et y encouragent la culture d'autres produits d'exportation sur de nouvelles bases technologiques ${ }^{(1)}$. Pour une série de raisons, le soja offre, dans le Mato Grosso, des résultats très favorables en terme de production. D'abord planté à l'essai dans des municipalités du sud-est de l'État actuel ${ }^{(2)}$, comme Rondonópolis et Primavera do Leste (aujourd'hui reconverties dans la production de graines), il a été étendu vers le Nord le long de l'axe de la route BR-163, qui relie la capitale Cuiabá à Santarém, dans l'État de Pará.

\section{Aire cueillie (ha) de soja en graines - Brésil, grandes régions}

\begin{tabular}{|c|c|c|c|c|c|c|c|c|c|c|c|}
\hline \multicolumn{2}{|c|}{$\begin{array}{l}\text { Grandes } \\
\text { régions }\end{array}$} & 1965 & 1970 & 1975 & 1980 & 1985 & 1990 & 1995 & 2000 & 2005 & 2007 \\
\hline BR & Brésil & 431834 & 1318809 & 5824492 & 8774023 & 10153405 & 11487303 & 11675005 & 13656771 & 122948874 & 20565279 \\
\hline co & $\begin{array}{l}\text { Région } \\
\text { Centre- } \\
\text { Ouest }\end{array}$ & 804 & 13693 & 249880 & 1130093 & 2844222 & 3810153 & 4531856 & 5530455 & 510854209 & 9014157 \\
\hline NE & $\begin{array}{l}\text { Région } \\
\text { Nord-Est }\end{array}$ & 511 & 16 & 757 & 1986 & 72217 & 376814 & 571085 & 847076 & 1441161 & 1452880 \\
\hline $\mathbf{N}$ & $\begin{array}{l}\text { Région } \\
\text { Nord }\end{array}$ & - & - & - & 85 & 39137 & 30920 & 24617 & 71960 & 514246 & 454285 \\
\hline SE & $\begin{array}{l}\text { Région } \\
\text { Sud-Est }\end{array}$ & 6349 & 63731 & 467197 & 723156 & 944156 & 1119587 & 1130655 & ; 1135064 & 41900077 & 1360955 \\
\hline s & $\begin{array}{l}\text { Région } \\
\text { Sud }\end{array}$ & 424170 & 1241369 & 5106658 & 6918703 & 6253673 & 6149829 & 5416792 & ? 6072216 & 8239181 & 8283002 \\
\hline
\end{tabular}

Source : IBGE - Produção Agrícola Municipal

\section{Aire cueillie (ha) de soja en graines - Brésil, grandes régions}

\begin{tabular}{|c|c|c|c|c|c|c|c|c|c|c|c|}
\hline \multicolumn{2}{|c|}{$\begin{array}{l}\text { Grandes } \\
\text { régions }\end{array}$} & 1965 & 1970 & 1975 & 1980 & 1985 & 1990 & 1995 & 2000 & 2005 & 2007 \\
\hline BR & Brésil & 431834 & 1318809 & 5824492 & 8774023 & 10153405 & 11487303 & 11675005 & 13656771 & 22948874 & 20565279 \\
\hline co & $\begin{array}{l}\text { Région } \\
\text { Centre- } \\
\text { Ouest }\end{array}$ & 804 & 13693 & 249880 & 1130093 & 2844222 & 3810153 & 4.531856 & 5530455 & 10854209 & 9014157 \\
\hline NE & $\begin{array}{l}\text { Région } \\
\text { Nord-Est }\end{array}$ & 511 & 16 & 757 & 1986 & 72217 & 376814 & 571085 & 847076 & 1441161 & 1452880 \\
\hline $\mathbf{N}$ & $\begin{array}{l}\text { Région } \\
\text { Nord }\end{array}$ & - & - & - & 85 & 39137 & 30920 & 24617 & 71960 & 514246 & 454285 \\
\hline SE & $\begin{array}{l}\text { Région } \\
\text { Sud-Est }\end{array}$ & 6349 & 63731 & 467197 & 723156 & 944156 & 1119587 & 1130655 & ; 1135064 & 1900077 & 1360955 \\
\hline s & $\begin{array}{l}\text { Région } \\
\text { Sud }\end{array}$ & 424170 & 1241369 & 5106658 & 6918703 & 6253673 & 6149829 & 5416792 & 6072216 & 8239181 & 8283002 \\
\hline
\end{tabular}


La production de la région Sud, qui en 1965 représentait presque la totalité de la production du pays, poursuit sa croissance tout au long des décennies qui ont vu la production diminuer dans d'autres régions. Il a fallu attendre 2005 pour que son aire cueillie soit dépassée par celle du Centre-Ouest. Dans le Centre-Ouest, le grand producteur de soja est l'État du Mato Grosso, qui possède à lui seul plus de la moitié de la production de toute la région, suivi par les États du Rio Grande do Sul et du Paraná.

\section{Soja et café, les produits phares de l'agrobusiness}

Dans le Minas Gerais, dans la région du triangle Mineiro, le succès du soja, produit encore aujourd'hui prédominant dans des municipalités comme Iraí de Minas, Uberaba et Uberlândia, est allé de pair avec des bons résultats d'autres produits, tels que le café dans la sous-région d'Alto Paranaíba ${ }^{(3)}$. Le café a une histoire plus longue et connue. Le Brésil reste le principal producteur mondial et jusqu'au début des années 2000 cette denrée se trouvait en tête de la liste des exportations agricoles du pays. Depuis, bien que dépassée par d'autres produits, sa production n'a cessé de croître.

Cultivé depuis plus d'un siècle dans un État qui, pendant la deuxième moitié du $\mathrm{XX}^{\mathrm{e}}$, comprend la région montagneuse du sud du Minas Gerais parmi les plus productrices du pays, le café est introduit dans la région d'Araguari en $1972^{(4)}$. Les nouvelles conditions de production créées par les programmes d'occupation des plaines du cerrado - correction de sols, irrigation, usage de pesticides, engrais chimiques et machines - ouvrent des perspectives très favorables à la culture du café d'appellation d'origine contrôlée destiné à l'exportation, le dénommé "café do cerrado". S'imposant rapidement dans la région, les résultats sont déjà encourageants au cours des années soixante-dix, dans les municipalités autour de Patrocínio et Araguari, atteignant son point culminant entre 1990 et 2000. Aujourd'hui, il s'y heurte à la concurrence de produits tels que la canne à sucre, mais conserve toujours une position importante dans l'économie, mobilisant des capitaux importants et affichant une croissance démographique remarquable.

Ces zones d'expansion de ce qui est devenu connu, au Brésil, sous le terme d'“agrobusiness" constituent des régions de forte croissance démographique. Bien que, comme le soulignait Georges Martine dans les années quatre-vingt à propos des frontières agricoles de l'époque, le volume de populations, en termes absolus, ne constitue pas une espèce de soupape d'échappement démographique 
pour ceux qui rêvent des grandes villes ; en termes relatifs, on note une transformation significative. Mais il n'y a pas d'homogénéité entre les différentes régions, ni d'expansion linéaire.

La comparaison entre deux régions particulièrement associées à la culture du soja et du café, puisque ces deux produits y occupent une place importante dans l'ensemble des denrées agricoles cultivées, permet de comprendre le sens de cette dynamique démographique. Nous avons choisi la zone de production de soja autour de la route BR-163, au Mato Grosso, et celle du

café do cerrado, dans le triangle Mineiro/Alto Paranaíba, au Minas Gerais ${ }^{(5)}$. La première est une zone d'occupation considérée comme récente et dédiée à la culture presque exclusive du soja, et la deuxième une aire d'occupation séculaire et de cultures diversifiées ${ }^{(6)}$.

Le contraste entre les taux de croissance démographique de l'ensemble du pays, des

Ces zones d'expansion de ce qui est devenu connu, au Brésil, sous le terme d'“agrobusiness" constituent des régions de forte croissance démographique. deux États où se développe l'agrobusiness et des régions qui sont l'objet de notre étude donne une idée des transformations démographiques associées à une entreprise de ce type.

Les choses deviennent encore plus claires lorsque nous descendons à une échelle locale : les municipalités autour desquelles nous avons concentré nos recherches.

\section{Variations de la population totale - Brésil, États et micro-régions - 1970-2000}

\begin{tabular}{|c|c|c|c|c|c|c|c|}
\hline \multirow[b]{2}{*}{$\begin{array}{l}\text { Brésil, État, } \\
\text { Meso et Micro- } \\
\text { régions }\end{array}$} & \multicolumn{4}{|c|}{ Population Totale } & \multicolumn{3}{|c|}{ Taux annuel de croissance } \\
\hline & 1970 & 1980 & 1990 & 2000 & $1970-1980$ & 1980-1991 & 1991-2000 \\
\hline Brésil & 93134846 & 119011052 & 146825475 & 157070163 & $2,5 \%$ & $1,9 \%$ & $0,8 \%$ \\
\hline Mato Grosso & 598849 & 1138918 & 2027231 & 2505245 & $6,6 \%$ & $5,4 \%$ & $2,4 \%$ \\
\hline $\begin{array}{l}\text { Meso-région } \\
\text { Mato Grosso Nord }\end{array}$ & 14195 & 164472 & 530699 & 709128 & $27,8 \%$ & $11,2 \%$ & $3,3 \%$ \\
\hline $\begin{array}{l}\text { Micro-région } \\
\text { d'Alto Teles Pires }\end{array}$ & 5692 & 13441 & 50839 & 101937 & $9,0 \%$ & $12,9 \%$ & $8,0 \%$ \\
\hline Minas Gerais & 11485663 & 13380105 & 15743152 & 17905135 & $1,5 \%$ & $1,5 \%$ & $1,4 \%$ \\
\hline $\begin{array}{l}\text { Meso-région } \\
\text { Triangle Mineiro / } \\
\text { Alto Paranaíba }\end{array}$ & 1095164 & 1337305 & 1595648 & 1871237 & $2,0 \%$ & $1,6 \%$ & $1,8 \%$ \\
\hline $\begin{array}{l}\text { Micro-région } \\
\text { d'Uberlândia }\end{array}$ & 271741 & 408844 & 564691 & 702074 & $4,2 \%$ & $3,0 \%$ & $2,4 \%$ \\
\hline
\end{tabular}




\section{Variations de la Population totale - États, micro-régions et municipalités - 1970-2000}

\begin{tabular}{|c|c|c|c|c|c|c|c|}
\hline \multirow[b]{2}{*}{$\begin{array}{l}\text { États, } \\
\text { Micro-régions, } \\
\text { Municipalités }\end{array}$} & \multicolumn{4}{|c|}{ Population Totale } & \multicolumn{3}{|c|}{ Taux annuel de croissance } \\
\hline & 1970 & 1980 & 1990 & 2000 & $1970-1980$ & 1980-1991 & 1991-2000 \\
\hline Mato Grosso & 598849 & 1138918 & 2027231 & 2505245 & $6,6 \%$ & $5,4 \%$ & $2,4 \%$ \\
\hline $\begin{array}{l}\text { Micro-région } \\
\text { d'Alto Teles Pires }\end{array}$ & 5692 & 13441 & 50839 & 101937 & $9,0 \%$ & $12,9 \%$ & $8,0 \%$ \\
\hline $\begin{array}{l}\text { Lucas do Rio } \\
\text { Verde }\end{array}$ & * & * & 6693 & 19316 & * & * & $12,5 \%$ \\
\hline Sorriso & * & * & 16107 & 35605 & * & * & $9,2 \%$ \\
\hline Minas Gerais & 11485663 & 13380105 & 15743152 & 17905135 & $1,5 \%$ & $1,5 \%$ & $1,4 \%$ \\
\hline $\begin{array}{l}\text { Micro-région } \\
\text { d’Uberlândia }\end{array}$ & 271741 & 408844 & 564691 & 702074 & $4,2 \%$ & $3,0 \%$ & $2,4 \%$ \\
\hline Araguari & 63368 & 83519 & 91283 & 101974 & $2,8 \%$ & $0,8 \%$ & $1,2 \%$ \\
\hline $\begin{array}{l}\text { Micro-région } \\
\text { de Patrocínio }\end{array}$ & 114239 & 126293 & 155905 & 183869 & $1,0 \%$ & $1,9 \%$ & $1,8 \%$ \\
\hline Patrocínio & 35578 & 44376 & 60753 & 73278 & $2,2 \%$ & $2,9 \%$ & $2,1 \%$ \\
\hline
\end{tabular}

Source : IBGE - Recensements démographiques de 1970, 1980, 1991 et 2000.

$\left(^{\star}\right)$ La municipalité n'avait pas encore été créée.

On peut constater, par exemple, à Sorriso et Lucas do Rio Verde, que ces centres urbains créés par la production de soja dans les années soixante-dix et quatre-vingt et convertis en municipalités au début des années quatre-vingt-dix, affichent des taux de croissance annuelle de la population bien supérieurs à ceux de l'État et du pays.

\section{Développement économique et croissance démographique : le rôle des migrations}

Cette croissance démographique est due, en grande partie, aux migrations. Dans le cas du Mato Grosso, cela va, pour ainsi dire, de soi, si l'on tient compte de l'infime quantité de personnes qui, au début des années soixante-dix, peuplaient une région ultérieurement consacrée au soja.

Pourtant, dans le cas du triangle Mineiro, une région occupée depuis longtemps et économiquement diversifiée, le phénomène n'est pas si explicite. Lorsque nous restreignons notre champ de comparaison aux seules activités agricoles, et plus spécifiquement au café, nous vérifions que sur une base territoriale bien moindre et avec des populations identiques à celles trouvées dans les municipalités d'Alto Teles Pires, les migrants ont un poids significatif. À Araguari, par exemple, $60 \%$ 
des 2718 personnes qui travaillaient en 2000 dans la culture du café venaient d'un État autre que le Minas Gerais.

Lanalyse des données statistiques montre que, dans les deux cas, cette hausse démographique n'est pas seulement liée aux mouvements migratoires en général, mais plus précisément aux mouvements migratoires provenant d'autres régions du pays, notamment la région Sud. Les individus originaires du Paraná, suivis de ceux du Rio Grande do Sul et de Santa Catarina, dans la région de Mato Grosso, et par les natifs de São Paulo, dans les municipalités étudiées du triangle Mineiro, sont majoritaires dans les deux cas.

La présence à Sorriso et Lucas do Rio Verde de migrants venus d'États d'autres régions, d'autres municipalités, ou même d'autres zones du Mato Grosso, ne s'accroît qu'à partir des années quatre-vingt-dix, avec l'établissement d'un nombre important de personnes originaires du Nord-Est, notamment du Maranhão. Dans les municipalités du triangle, la migration de natifs du Paraná et de São Paulo accompagne pari passu celle des originaires du Nord-Est, notamment les baianos (quoique moins hégémoniques que les natifs du Maranhão à Mato Grosso). Les deux phénomènes atteignent leur apogée dans les années quatre-vingt-dix ${ }^{(7)}$.

\section{Origine des migrants dans les municipalités de Soriso et Lucas do Rio Verde - Mato Grosso - $1995-2000$}

\begin{tabular}{|c|c|c|c|c|}
\hline $\begin{array}{l}\text { État de Résidence } \\
\text { au 31/07/2000 }\end{array}$ & $\begin{array}{l}\text { Lucas do Rio } \\
\text { Verde }\end{array}$ & & Sorriso & \\
\hline $\begin{array}{l}\text { Population totale } \\
\text { Nés après } 1995\end{array}$ & 19316 & $100 \%$ & 35604 & $100 \%$ \\
\hline Résidents dans l'État en 1995 & 2168 & $11 \%$ & 4016 & $11 \%$ \\
\hline $\begin{array}{l}\text { Non-résidents dans l'État } \\
\text { en 1995* }\end{array}$ & 13458 & $70 \%$ & 24563 & $69 \%$ \\
\hline État de résidence antérieure & 3690 & $19 \%$ & 7026 & $20 \%$ \\
\hline Paraná & 1148 & $\cdot$ & 1554 & \\
\hline Rio Grande do Sul & 728 & & 866 & \\
\hline Santa Catarina & 440 & & 969 & \\
\hline Mato Grosso do Sul & 167 & & 768 & \\
\hline São Paulo & 157 & & 443 & \\
\hline Rondônia & 105 & & 176 & \\
\hline Pará & 113 & & 251 & \\
\hline Goiás & 78 & & 145 & \\
\hline Maranhão & 82 & & 208 & \\
\hline Autres États & 39 & & 306 & \\
\hline Non déterminé & 633 & & 1332 & \\
\hline
\end{tabular}

Source : IBGE - Recensement démographique de 2000

* Y compris ceux qui ont migré avant 1995 


\section{Origine des migrants dans les municipalités d'Araguari et Patrocínio - Minas Gerais. 1995-2000}

\begin{tabular}{|l|r|r|}
\hline $\begin{array}{l}\text { État de Résidence } \\
\text { au 31/07/2000 }\end{array}$ & Araguari & Patrocínio \\
\hline Population totale & 101974 & 73278 \\
\hline Nés après 1995 & 8089 & 7165 \\
\hline Résidents dans l'État en 1995 & 93885 & 66113 \\
\hline Non-résidents dans l'État en 1995 * & & \\
\hline État de résidence antérieur & & \\
\hline Goiás & 1109 & 281 \\
\hline São Paulo & 863 & 686 \\
\hline Paraná & 611 & 382 \\
\hline Ceará & 495 & 37 \\
\hline Pará & 217 & 6 \\
\hline District Fédéral & 179 & 178 \\
\hline Santa Catarina & 135 & 20 \\
\hline Rio de Janeiro & 106 & 33 \\
\hline Mato Grosso do Sul & 103 & 40 \\
\hline Tocantins & 101 & 18 \\
\hline Maranhão & 77 & 307 \\
\hline Mato Grosso & 63 & 42 \\
\hline Pernambuco & 50 & 98 \\
\hline Bahia & 49 & 14 \\
\hline Rio Grande do Sul & 43 & \\
\hline & & \\
\hline
\end{tabular}

Source: IBGE - Recensement démographique de 2000

${ }^{*}-Y$ compris ceux qui ont migré avant 1995

\section{Provenance et ascendance comme critères du positionnement social}

Ainsi, ce n'est pas un hasard si la "carte sociale" élaborée par ceux qui vivent dans ces régions-là fait référence au lieu de naissance réel ou supposé des personnes nommées : "gaúchos" et "maranhenses" dans la zone du soja à Mato Grosso ; "paulistas" ou "paranaenses" et "baianos", en plus des "mineiros", dans la région caféière du triangle Mineiro ${ }^{(8)} \ldots$.. Les habitants des endroits étudiés utilisent ce type de classification pour raconter aux enquêteurs l'histoire des lieux où ils habitent, pour se repérer dans leur quotidien ("aujourd'hui, j'ai discuté avec un maranhense"; " "à la fête il n'y avait que des gaúchos" ou "la fazenda appartient à un paranaense"; ; "jai rencontré un baiano à la station de bus"), ou même pour répertorier les quartiers de leur ville ("bairros de gaúcho", les plus valorisés, ou "bairros 
de maranhense", ceux aux conditions plus précaires, pour ne citer que le cas du Mato Grosso), fondant ou confirmant une ségrégation géographico-sociale qui n'est pas sans impressionner les visiteurs. Cette ségrégation se manifeste également dans les images que les "sudistes" se font des maranhenses (ou, dans le Minas Gerais, les paranaenses à propos des baianos) : à leurs yeux, ces derniers concentrent tous les vices et sont responsables de la violence qui serait en train de gagner les villes de la région, sans qu'on ne trouve d'accusations aussi exacerbées dans la direction opposée.

Dans les zones de culture prédominante du soja du triangle Mineiro, comme Iraí de Minas, la municipalité où se sont établis les premiers colons venus du Sud, soutenus par les programmes gouvernementaux des années soixante-dix, "gaúcho" est employé comme un terme définitoire avec un sens similaire à celui du Mato Grosso. La même chose semble se produire dans les municipalités plus au nord où, associés à la culture du soja et d'autres produits, des migrants du Sud sont ainsi désignés ${ }^{(9)}$. Pourtant, dans l'ensemble du triangle Mineiro, où les paranaenses, comme à Alto Teles Pires, constituent le plus grand nombre, surtout dans les aires caféières, ce sont les termes "paranaense" ou "paulista" qui désignent ceux qui viennent du Sud ${ }^{(10)}$.

\section{Les réseaux de parenté des migrants}

Pour tous ceux qui sont arrivés du sud du pays, ce déplacement pour le Mato Grosso ou d'autres États ne constituait pas une première. La plupart d'entre eux, pour ne pas dire la presque totalité, avait une expérience personnelle, à la tête de leurs familles ou, pour les plus jeunes, avec leurs parents, de déplacements précédents à l'intérieur de la région Sud et/ou de déplacements dans l'État où ils habitent à présent. Du fait de ces déplacements, collectifs ou individuels, mais toujours organisés par la famille, certains des individus résidant actuellement dans la région étudiée ont laissé des parents plus ou moins proches (père et mère, enfants, frères ou sceurs, oncles, cousins...) dans leurs lieux d'origine et aussi dans les régions traversées. Ces réseaux s'étendent parfois à des zones plus lointaines, encore en processus d'intégration à la production agricole "moderne". Les relations avec ces parents sont entretenues de plusieurs manières, notamment par les visites dans la période entre les récoltes ou à l'occasion des fêtes de fin d'année. Ces mécanismes, conçus dans le passé, continuent d'être actionnés et conditionnent les décisions de déménagement. La perspective de déplacement semble être 
aussi essentielle à la planification de la reproduction sociale de ces familles que l'existence de terres permettant d'exercer leur activité productrice. Loin d'être une possibilité envisagée seulement en cas de situations limite, l'éventualité de déplacement, là où les premiers à arriver peuvent avoir accès à une parcelle de terre ou à une parcelle plus grande, est évaluée quotidiennement ${ }^{(11)}$. Même ceux qui se déplacent en tant que travailleurs salariés obéissent, pour la plupart, à une stratégie familiale et ne perdent pas de vue l'objectif de devenir propriétaire d'une parcelle de terre, là où le prix de la terre sera moins cher.

\section{Des interprétations différentes de la possession du territoire}

Quoique également orientés par des stratégies familiales, les déplacements de maranhenses vers le Mato Grosso et de baianos vers Minas Gerais suivent une dynamique différente. Ni les municipalités d'Alto Teles Pires ni celles du triangle Mineiro ne constituent un point d'arrivée pour les uns ou les autres. L'acquisition d'une parcelle n'est pas un objectif majeur. Pour beaucoup de familles, l'infrastructure routière des zones d'agrobusiness représente un moyen d'accès au marché du travail dans des aires agricoles ou agro-industrielles du Centre, du Sud-Est ou du Sud. Ces familles parcourent un trajet ponctué de travaux temporaires dans diverses régions du pays, accompagnant la récolte de différents produits, et reviennent à la fin à leur lieu d'origine. Ainsi, des maranhenses qui travaillent dans la récolte du soja dans le Mato Grosso, passent à celle du café dans le triangle Mineiro, se déplacent ensuite à São Paulo

Les fazendas de soja et de café sont des territoires de passage, même lorsque ce passage se prolonge. ou dans le Paraná pour la coupe de la canne à sucre ou la récolte du coton, et finalement retournent dans le Maranhão, recommençant le cycle tous les ans. La même chose se produit pour les baiano qui travaillent avec une certaine régularité dans la récolte du café dans le Minas Gerais et qui, à la fin du cycle agricole de ce produit, effectuent un itinéraire similaire à celui des maranhenses, avant de retourner à leurs fermes dans le Nord-Est. Tant dans le cas des maranhenses que dans celui des baianos, et même si certains finissent par s'établir dans le BTP ou le petit commerce dans les régions où ils se déplacent à la recherche de travail, le modèle prédominant demeure celui où la maison familiale se trouve dans l'État d'origine. 
De cette façon, la perspective d'un travail dans une région plus proche de la famille et/ou de l'offre de salaires plus élevés peut transformer ce long parcours en un mouvement d'allers-retours entre la zone de résidence et la région choisie comme lieu de travail, à l'image de ce qui semble se produire actuellement dans le Mato Grosso. Les ressources obtenues grâce au travail dans les fazendas de Mato Grosso ne sont pas utilisées, dans ce cas précis, pour l'achat de terre ou d'autres biens qui 
permettraient aux maranhenses de s'installer dans cet État, mais canalisées, au moins idéalement, vers la consolidation de la position de leurs familles dans le Maranhão, ce qui peut inclure l'acquisition de terre ou de bétail, ou simplement la pérennisation du mode de consommation de ces familles ${ }^{(12)}$.

En admettant l'existence d'un conflit ou d'une dispute entre gaúchos et maranhenses dans le Mato Grosso autour du soja ou entre paranaenses/paulistas et baianos dans le Triangle autour du "café do cerrado", nous pourrions affirmer être devant une "dispute imparfaite". Il ne s'agit pas d'une dispute entre groupes asymétriques (comme ils le sont effectivement) pour un même objet. Ce qui existe bel et bien, c'est une dispute de "territoire", comme on l'a vu dans la ségrégation spatiale, mais ce sont des territoires que les stratégies familiales des uns et des autres interprètent différemment.

Les modalités de présence sont distinctes. Les "sudistes", dans les deux cas, veulent s'établir, et le font, comme s'ils étaient chez eux. De façon symptomatique, dans le Mato Grosso, ils se définissent comme ceux "d'ici", "de la terre" et dans le triangle du café, bien que cette identification continue d'être liée aux mineiros, on trouve des exemples de paranaenses qui réclament cette condition. Mais le monde et le lieu des natifs du Nord-Est est tout autre : il est là où se trouvent leurs familles. Les fazendas de soja et de café sont des territoires de passage, même lorsque ce passage se prolonge. On est donc en présence de deux groupes en mouvement permanent (maranhenses et baianos ne sont pas plus sédentaires que les "sudistes") qui se heurtent.

\section{Blbliographie}

- Andrade Maristela de Paula, Os Gaúchos descobrem o Brasil. Projetos Agropecuários contra a Agricultura Camponesa, São Luiz, EDUFMA, 2008.

- Araujo N.B., I. Wedekin, L.A. Pinazza, Complexo Agroindustrial - Agribusiness Brasileiro, São Paulo. Agroceres, 1990.

- Bernardes Julia Adão, "Circuitos espaciais da produção na fronteira agrícola moderna: BR-163 matogrossense", in J.A. Bernardes e O.L. Freire Filho, Geografias da Soja BR-163. Fronteiras em Mutaçấo, Rio de Janeiro, Ed. Arquímedes, 2005.

- Cardoso de OliveiraLuis, Colonizaçâao e diferenciaçâa : os colonos de Canarana, mémoire de Master, PPGAS/MN/UFRJ, 1993.

- Chaves Christine, Festas da Política. Uma etnografia da modernidade no sertáo (Buritis-M). Rio de Janeiro, Relume Dumará, 2003.

- Haesbaert Rogério, Dês-territorialização e identidade. A rede gaúcha no NE, Niteroi, Ed. UFF, 1997.

- IBGE, "Produção Agrícola Municipal", Censos 1970-2000, 2004.

- Martine George, "O significado demográfico da fronteira agrícola", Espaço e Debates, Ano IV, no 13, 1984.

- Monbeig Pierre, Pioneiros e fazendeiros de Sáo Paulo, São Paulo, Hiucitec/Polis, (1977), 1984. 
- Novaes Roberta Brandão, Forasteiros: Trajetórias, experiências de trabalho e práticas de deslocamento dos trabalhadores do café no Alto Paranaíba, mémoire de Master, Rio de Janeiro, CPDA/UFFRJ, 2009.

- Pinheiro Souza Junior Haiton, O lugar do progresso: famílias, trabalho e sociabilidade em uma comunidade de produtores de café do Cerrado Mineiro, mémoire de Master, Rio de Janeiro, PPGSA/IFCS/UFRJ, 2009.

- Maria Sylvia Macchione Saes, "O agronegócio café do Brasil no mercado internacional", Revista FAE Business, no 9, setembro, 2004.

\section{Notes}

1. Ces zones ont fait l'objet de diverses politiques publiques visant le développement d'une agriculture tournée vers le marché dans le cerrado brésilien, à travers différents programmes du gouvernement fédéral et de l'État au début des années soixante-dix, lesquels se sont succédés tout au long de la décennie. Le POLOCENTRO, programme créé en 1975 et qui avait pour but d'accélérer l'occupation rationnelle des zones de cerrado, a été remplacé, en 1979, par le PRODECER et ses nombreux programmes subsidiaires.

2. Dans les années soixante-dix, l'ancien État du Mato Grosso n'avait pas encore été divisé en deux États : le Mato Grosso correspond au Nord de l'état primitif et le Mato Grosso do Sul à sa partie sud. La scission a eu lieu le $1^{\text {er janvier } 1979 .}$

3. Dans les dix dernières années, l'importance d'autres produits dans d'autres municipalités de la région s'est significativement accrue. Aux expériences de reforestation déjà en cours sont venues s'ajouter les plantations de vergers et de potagers, la culture de la canne à sucre, en plus du traditionnel maïs, maintenant cultivé sous de nouvelles formes, ainsi que la modernisation de l'élevage, lui aussi traditionnellement pratiqué.

4. Celia R.G. Olivaira, A frente cafeicultora em Araguari- $M G$, mémoire de Master. São Paulo, FFLECH, Universidade de São Paulo, 1991.

5. Les recherches qui ont donné lieu à cet article font partie d'un projet plus grand - "Société et économie de l'agrobusiness " - développé par l'UFRJ et l'UNFFRJ, coordonné par Beatriz Herédia, Leonilde Medeiros, Moacir Palmeira et Sérgio Pereira Leite, avec la participation de 19 chercheurs. Le projet reçoit le soutien financier de la Fondation Ford, du CNPq et de la FAPERJ. Outre des études ethnographiques, la recherche inclut une étude socio-économique aux niveaux macro et micro et l'analyse des institutions et des acteurs politiques impliqués.

6. L'étude a été menée de façon exhaustive dans ces régions, mais dans le cadre de cet article, nous manierons surtout des données relatives aux municipalités où siègent les principales agglomérations urbaines : Sorriso et Lucas do Rio Verde pour le soja, dans la micro-région d'Alto Teles Pires, dans le Mato Grosso ; et Patrocínio et Araguari pour le café, dans la meso-région du Triangle Mineiro / Alto Paranaíba, dans le Minas Gerais.

7. Recensement démographique de 2000.

8. Cette classification sociale des personnes selon leur lieu de naissance ou d'origine avait déjà été signalée par Pierre Monbeig dans les régions d'expansion du café de l'État de São Paulo à la fin des années trente. Ici, à la distinction entre paulistas, identifiés à la culture du café et mineiros, associés à l'“agriculture d'autosubsistance" et à l'élevage porcin, qui avait précédé la plantation des caféiers, s'ajoutera celle entre paulistas, considérés comme les grands producteurs de café, et baianos, terme utilisé pour désigner ceux qui travaillaient dans le café, dans leur majorité originaires du Nord-est.

9. La catégorie gaúcho a déjà retenu l'attention de plusieurs chercheurs : Maristela Andrade pour le Maranhão ; Rogério Haesbaert pour Bahia ; Christine Chaves pour le Minas Gerais et, précédemment, José Vicente Tavares dos Santos et Luís Roberto Cardoso de Oliveira pour le Mato Grosso.

10. Cette affirmation est surtout valable pour les municipalités sur lesquelles se concentre notre analyse. Puisque, en termes quantitatifs, on constate des variations importantes entre les municipalités du triangle, où la fréquence de natifs de l'État voisin de Goiás est remarquable, ainsi que la forte présence de personnes nées dans des municipalités d'autres régions du Minas Gerais, il serait convenable de perfectionner cette analyse afin de pouvoir faire des affirmations plus génériques.

11. Cristiano Desconsi, A Marcha dos "pequenos" proprietários rurais no Mato Grosso: um estudo a partir da trajetória de migrantes do sul do Brasil para a microrregiäo de Alto Teles Pires, mémoire de Master, Rio de Janeiro, CPDA/UFRRJ, 2009.

12. Ariana Rumstain, Peóes no trecho: estratégias de deslocamento de trabalhadores no Mato Grosso, mémoire de Master, Rio de Janeiro, PPGAS/MN/UFRJ, 2009. 